\title{
基于第一性原理计算的纳米氧化铇研究进展
}

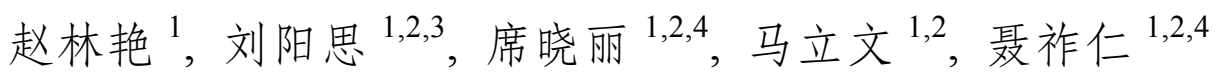

(北京工业大学 1. 材料与制造学部 新型功能材料教育部重点实验室; 2. 工业大数据应用技术国家工程实验室; 3. 北 京古月新材料研究院; 4. 首都资源循环材料技术省部共建协同创新中心，北京 100124)

摘 要: 纳米氧化铇作为一种具有独特物理化学性质的半导体功能材料, 已被广泛应用于环境、能源、生命科学、 信息技术等领域。本文基于第一性原理计算在纳米氧化铇中的应用进展, 概述了量子力学基础上的第一性原理及密 度泛函理论的发展历程及基本理论，介绍了该领域常用的 MS (Materials studio)、VASP (Vienna ab initio simulation package)等模拟计算软件, 并分类阐述了第一性原理计算对氧化铇的微观电子结构、物质相互作用、分子热动力学 等方面的研究成果。最后提出了第一性原理计算在纳米氧化铇这类半导体材料研究中存在的问题及未来发展趋势。 关 键 词: 纳米氧化钨; 第一性原理; 模拟计算; 电子结构; 综述

中图分类号: TB34 文献标志码: A

\section{First-principles Study on Nanoscale Tungsten Oxide: a Review}

\author{
ZHAO Linyan ${ }^{1}$, LIU Yangsi ${ }^{1,2,3}$, XI Xiaoli ${ }^{1,2,4}$, MA Liwen ${ }^{1,2}$, NIE Zuoren ${ }^{1,2,4}$
}

(1. Key Laboratory of Advanced Functional Materials, Ministry of Education, Faculty of Materials and Manufacturing, Beijing University of Technology, Beijing 100124, China; 2. National Engineering Laboratory for Industrial Big-data Application Technology, Beijing University of Technology, Beijing 100124, China; 3. Beijing GUYUE New Materials Research Institute, Beijing University of Technology, Beijing 100124, China; 4. Collaborative Innovation Center of Capital Resource-Recycling Material Technology, Beijing University of Technology, Beijing 100124, China)

\begin{abstract}
Nanoscale tungsten oxide, as a functional semiconductor with unique physical and chemical properties, is widely used in environment, energy, life science and information technology fields. Based on the application of first-principles study in nanoscale tungsten oxide, the functions of theory calculations are reviewed in the paper. Firstly, the development and basic theory of the first principles and density functional theory are illustrated based on quantum mechanics. Then, the commonly related software in such field of semiconductors, such as MS (Materials Studio) and VASP (Vienna $a b$ initio Simulation Package) are introduced. Furthermore, the recent study of the first-principles on tungsten oxide in terms of electronic structure, interaction of materials, molecular thermodynamics, and so on, is clarified. Finally, the existing problems and future developments of theory calculations used in the field are summarized and prospected.
\end{abstract}

Key words: nanoscale tungsten oxide; first-principles; theory calculations; electronic structure; review

铇 $(\mathrm{W})$ 是一种过渡金属元素, 在其氧化物中, 成化学计量比的三氧化铇 $\left(\mathrm{WO}_{3}\right)$ 是一种半导体材料,

收稿日期：2020-11-28; 收到修改稿日期：2021-04-26; 网络出版日期：2021-06-01

基金项目: 国家重点研发项目(2018YFC1901700); 国家自然科学基金(51621003, 51702008); 北京自然科学基金(2202010) National Key R\&D Program of China (2018YFC1901700); National Natural Science Foundation of China (51621003, 51702008); Beijing Natural Science Foundation (2202010)

作者简介：赵林艳(1992-), 女，博士研究生. E-mail: zlyding@emails.bjut.edu.cn ZHAO Linyan(1992-), female, PhD candidate. E-mail: zlyding@emails.bjut.edu.cn

通信作者：席晓丽，教授.E-mail: xixiaoli@bjut.edu.cn. XI Xiaoli, professor. E-mail: xixiaoli@bjut.edu.cn 
具有常温稳定、气敏性好、储电量大等优异性能 ${ }^{[1-3]}$, 受到研究者广泛关注; 而非化学计量比的 $\mathrm{WO}_{3-x}$, 因含有氧空位缺陷, 被认为具有不同于 $\mathrm{WO}_{3}$ 的独特 物理化学性质, 近年来也越来越受到关注 ${ }^{[4-5]}$ 。综合 本课题组及其他学者对氧化铇的研究 ${ }^{[6-8]}$, 本文总 结列出了几种常见氧化铇的晶型结构、空间分布及 $3 \mathrm{D}$ 模型(表 1)。

人们对氧化铇的研究最早可追溯到 17 世纪, 人 们首先研究了 $\mathrm{WO}_{3} 、 \mathrm{NaWO}_{3}$ 及 $\mathrm{LiWO}_{3}$ 等人工合成 的铇氧化物 ${ }^{[9]}$ 。相对于 $\mathrm{TiO}_{2} 、 \mathrm{ZnO} 、 \mathrm{NiO}$ 等同类材 料, 氧化铇的禁带宽度更窄 ${ }^{[10-11]}$, 是一种廉价而稳

表 1 不同晶型氧化铇的空间结构及 3D 模型

Table 1 Tungsten oxides with different crystal structures, space groups and $3 \mathrm{D}$ models ( $\mathrm{O}$ and $\mathrm{W}$ atoms are represented by red and blue balls, respectively)

\begin{tabular}{cc}
\hline Type of tungsten oxide & Configuration \\
\hline Cubic $\mathrm{WO}_{3}$ & $\mathrm{pm} 3 \mathrm{~m}(221)$ \\
Hexagonal $\mathrm{WO}_{3}$ & $\mathrm{p} 6 / \mathrm{mmm}(191)$ \\
& $\mathrm{p} 4 / \mathrm{ncc}(130)$ \\
& $\mathrm{p} 2 / \mathrm{m}(10)$ \\
Tetragonal $\mathrm{WO}_{3}$ & $\mathrm{pl}(1)$ \\
Orthorhombic $\mathrm{WO}_{3}$ & $\mathrm{p} 2 / \mathrm{c}(14)$
\end{tabular}

定的 $\mathrm{n}$ 型半导体过渡金属氧化物，在气敏 ${ }^{[12]}$ 、光催 化 $^{[13]}$ 、光致变色 ${ }^{[14]}$ 、电致变色 ${ }^{[15]}$ 、锂离子电池 ${ }^{[16]}$ 及疾病治疗 ${ }^{[17]}$ 等领域应用广泛。

本课题组在对氧化铇的研究中发现, 纳米级氧 化铇在光催化降解有机染料及微污染物方面表现优 异 ${ }^{[18-20]}$, 有望在环保领域得到广泛应用。与大尺寸 的氧化铇相比, 纳米尺度的氧化铇具有可调控的表 面能、显著增大的比表面积以及量子限域效应等优 点 ${ }^{[21-22]}$, 因此目前对纳米氧化铇的研究也逐渐成为 热点，其在清洁能源、信息技术、环境科学等领域 均拥有巨大的应用潜力。在这些前沿领域中, 基于 密度泛函理论(Density functional theory, DFT)的第 一性原理(First-principles)计算与实验数据相结合的 分析方式已经成为重要的研究手段。第一性原理计 算在一定程度上可以节约人力物力, 减少资源浪费, 具有降低成本、绿色环保、准确可控、“前瞻性”和“创 新性”等优点 ${ }^{[23-26]}$ 。利用其开展的研究大致分为三个 方面: 1)辅助实验研究: 当实验或检测条件苛刻、不 易实施时，可通过理论计算辅助解决问题，降低成 本的同时获得所需性质；2)指导实验方向：模拟实 验过程, 揭示实验现象背后的微观本质, 指导人们 较快地开展相关方面研究; 3) 设计预测新材料: 预 测并解释新材料的性能, 提升新材料设计的可行性 及准确性。

目前, 纳米尺度下氧化铇材料研究面临的主要 问题有 ${ }^{[24]}$ : 1) 实验重复性差, 2)复杂或苛刻条件下的 实验或测试难以开展, 3) 实验研究侧重大量纳米结 构的共同作用结果，对单一结构的作用机理无法考 证，4)由于氧化铇材料表面的不均匀性和复杂性, 对材料表面具体问题的研究难以明确统一等。利用 第一性原理计算可以辅助解决上述部分问题, 如通 过模拟表面气体分子吸附与反应的微观作用, 揭示 氧化铇的气敏检测机理 ${ }^{[27]}$; 建立合理的催化剂表 面模型可以很好地研究氧化铇表面的水吸附和催化 产氢效率 ${ }^{[28]}$; 对氧化铇中掺杂元素进行初步篮选, 提高掺杂成功率、减少实验工作量等 ${ }^{[29]}$ 。

综合上述内容可以看出，第一性原理计算对纳 米氧化铇的制备工艺及性能研究具有重要意义。目 前, 将第一性原理计算应用于纳米氧化铇等半导体 材料的研究已渐成趋势, 但相关研究的总结及有关 发展规律和趋势尚未见综述加以论述。因此本文总 结了近年来第一性原理计算在纳米氧化铇半导体催 化剂领域的研究现状, 并对其未来发展方向作了适 当展望。 


\section{1 理论简介}

\section{1 第一性原理}

量子力学是现代物理学的重要基础, 也是 20 世 纪最伟大的发现之一 ${ }^{[30]}$ 。利用量子力学可以解释和 预测多种体系的物理化学性质, 并定量描述其电子 运动规律 ${ }^{[31]}$ 。然而在实际研究中, 考虑到多粒子系 统相互作用的复杂性, 求解 Schrödinger 方程非常困 难。由物理学的基本理论可知: 电子的质量比原子 核的质量小很多，但电子的运动速度却比原子核的 速度快千倍。由此, Born 和 Oppenheime ${ }^{[32]}$ 于 1927 年提出了绝热近似理论，即 Born-Oppenheimer(B-O) 近似理论。该理论将包含多电子、多原子核的原始 体系模型转化为更简单的多电子体系模型。在此基 础上, Hartree 与 Fock ${ }^{[33]}$ 进一步得出了对应单电子体 系模型的 Hartree-Fock 方程, 极大地简化了求解 Schrödinger 方程本征值的过程。

第一性原理正是基于量子力学的方法、从电子 运动角度出发而研究材料性质的一种计算方法 ${ }^{[34]}$ 。 广义理解上的第一性原理通常包括 $A b$ initio 从头 计算和 DFT 计算两种。其中 $A b$ initio 从头计算是 基于 Hartree-Fork(H-F)自洽场来计算的 ${ }^{[35]}$, 但 H-F 自洽场没有充分考虑电子之间的相互关系(目前所 说的从头算法是包括 H-F 方法及在此基础上引入 电子关联作用后校正的 H-F 方法的统称)。DFT 计 算 ${ }^{[36]}$ 是逐渐发展起来的计算理论, 其关键在于: 1)考 虑了电子交换及其相互关联效应；2)用电子密度代 替 $\mathrm{N}$ 电子波函数，相比从头算方法，计算量减少。 现代第一性模拟仿真中通常把基于 DFT 的方法叫 做第一性原理计算，也即狭义第一性原理。

\section{2 密度泛函理论}

波函数包含了系统所有信息，这大大限制了其 实际应用的范围，而 DFT 的确立解决了波函数复杂 性与庞大性的问题 ${ }^{[37]}$ 。DFT 的基本思想: 改变传统 以轨道波函数为基础的特点, 用粒子密度函数表述 系统基态各物理量, 以电子密度函数来表示体系能 量 ${ }^{[38]}$ 。通过成功采用各种有效的近似方法, DFT 用 单电子体系简化了多电子问题, 是电子结构与系统 总能量计算中不可缺少的重要理论 ${ }^{[37,39]}$ 。以下为近 似简化后的几种方法。

1) Thomas-Fermi-Dirac(T-F-D)近似

DFT 最初是在二十世纪六十年代前后, 在 Thomas- Fermi(T-F)理论的基础上发展起来的 ${ }^{[40]}$ 。 $\mathrm{T}-\mathrm{F}$ 理论首次提出以粒子密度为多电子体系研究对 象, 对 DFT 的发展具有重要作用，但其并未考虑电 子之间交换项的影响。1930 年 Dirac 以经验的方式,
将具有交换相互作用项的局域近似引入 $T-F$ 理论中, 形成了 T-F-D 理论 ${ }^{[41]}$ 。T-F-D 理论近似描述了一种 用于处理量子多体体系的新方法，但由于这种近似 忽略了体系中一些基本的物理化学本质现象, 如原 子轨道局域性的不同、分子中的成键等，因而不能 用于处理真实体系中的电子结构。

2) Hohenberg-Kohn(H-K) 定理

作为一个严格的多体体系理论，现代 DFT 建立 在两个著名的定理之上- - H-K 第一定理和第二定 理 ${ }^{[40]}$ 。1964 年, Hohenberg 和 Kohn 在研究 T-F 理论 模型时提出了这两个定理。H-K 定理中描述到: 多 粒子系统基态的基本变量可由粒子数密度函数来确 定，而系统基态的确定则是通过能量泛函对粒子数 密度变分实现的。该定理确定了相互作用多体系统 的基态能量与粒子密度之间的对应关系，是现代 DFT 最重要的基础之一。可是该定理框架下的能量 泛函表达式仍不够完善, 存在动能泛函项与交换相 互作用泛函项均无法确定的问题。

\section{3) Kohn-Sham(K-S)方程}

1965 年, Kohn 和 Sham ${ }^{[41]}$ 设想了一种非相互作 用的多电子体系，该体系与相互作用多电子体系具 有相同的电子密度，但把所有复杂的相互作用部分 放在了交换关联项中, 由这种多电子体系导出的电 子方程即为 K-S 方程。利用 K-S 方程求解密度泛函 的过程更简洁、结果更精密(通常所谓的自洽场方法 就是自洽求解 K-S 方程的过程, 需要指出的是, K-S 方程只是用来计算相互作用体系基态总能量的中间 步骤, 除了总能量有明确的物理意义外, 其它如本 征波函数、本征能量等并无真正的物理意义)。

\section{4) 交换关联泛函}

求解 K-S 方程时, 交换关联泛函项 $E_{\mathrm{xc}}[\rho]$ 总是 存在未知性和不确定性, 因此目前发展形成了 LDA (Local density approximation) $^{[42]}$ 、GGA (Generalized gradient approximation $)^{[43]}$ 及杂化泛函等多种交换关 联泛函近似方法来解决这一问题。LDA 以均匀电子 气体系的交换相互作用泛函近似，替代了非均匀体 系的泛函，在多数系统中都可以计算出相对可靠的 结果，但在描述真实体系时存在密度变化剧烈的问 题, GGA 克服了 LDA 的这一缺陷, 但使用 GGA 计 算后原始晶胞参数变化较大。杂化泛函则是一种把 $\mathrm{H}-\mathrm{F}$ 方法得到的交换能与密度泛函得到的交换能相 加, 以此得出体系交换能的方法, 选用适当的杂化 泛函后会使计算出的带隙和态密度等具有更高的可 信度, 但计算量较大。

需要指出的是, 在小分子及催化反应物/产物的 研究中, 由于涉及吸附、弱极性等弱相互作用, 长程 
范德华作用力的修正显得比较重要, 即理论计算中需 要考虑色散作用。然而很多交换关联泛函(如 B3LYP、 PBE、PW91)对色散作用的描述欠佳或完全不能描述, 此时就需要在计算过程中加入 DFT-D 色散矫正项 (目前常用 DFT-D3 矫正, 且有益无害), 不过部分杂 化泛函本身就已经考虑了色散修正(如 B97D、 B2PLYPD、 $\omega$ B97XD), 因此需注意避免重复矫正。

\section{3 噟势-平面波方法}

材料的性质往往取决于最外层的价电子, 由此 人们想到引入噟势的概念 (一种假想的势能), 把内 层电子与原子核作为整体(离子实)考虑, 用以代表 原子内的真实势能。引入噟势的优点: 减少展开系 统内层电子以及价电子区的波函数所需要的平面波 基组数，降低计算存储空间和所需的 CPU 时间。噟 势-平面波法 ${ }^{[44]}$ 就是通过构造噟势函数, 使得系统 外部区域的价电子赝势波函数与真实的物理波函数 具有相同性质的一种方法。常使用的赝势函数有超 软噟势 ${ }^{[45]}$ 、投影缀加波噟势 ${ }^{[46]}$ 以及模守恒噟势 ${ }^{[47]}$ 等。

\section{2 软件介绍}

在纳米氧化铇的研究中常用的第一性原理计算 相关软件, 有些属于商业软件, 功能强大, 易于维 护, 使用安全; 有些可免费获取, 操作简单, 易学 易懂, 使用率高。它们在纳米氧化铇类半导体材料 的研究中使用较广, 各具特色优势。

\subsection{Materials studio (MS)}

MS 材料模拟软件融合了多种时间和空间尺度 的模拟方法, 可实现从微观电子结构到宏观性能预 测的跨尺度科学研究, 是目前分子模拟领域中相对 精确、稳定和高效的材料模拟商用计算软件, 其以 Materials visualizer 为核心, 主要包括 CASTEP、 $\mathrm{DMol}^{3} 、$ Forcite+ 及 COMPASS 等共计 22 个模块 ${ }^{[48]}$ 。 Materials visualizer 给出视窗界面, 可用于构建模 型、调整参数和分析计算所得结果等, 并给出数据 图表进行动画演示。CASTEP 是 MS 软件中的重要 模块之一, 其功能主要包括结构优化、过渡态搜索、 反应速率计算和电子结构解析等, 目前已广泛应用 于半导体、陶瓷、金属、分子篮、各类晶体材料及 各种缺陷结构的模拟计算中。MS 的多模块计算特 点, 适用于纳米氧化铇各项性能的研究, 除上述 CASTEP 模块外, 还可利用 Forcite+模块研究氧化 铇的生长机制; 利用 $\mathrm{DMol}^{3}$ 模块可计算元素掺杂对 $\mathrm{WO}_{3}$ 催化剂的影响、不同构型氧化铇的稳定性等。

\subsection{Vienna $a b$ initio simulation package (VASP)}

VASP 是维也纳大学 Hafner 小组 ${ }^{[49]}$ 开发的一款
软件包, 是目前材料模拟和物质计算科学研究中较 流行的商用软件之一, 具有以下特点：1)既可求解 $\mathrm{K}-\mathrm{S}$ 方程, 也可以求解 Roothaan 方程; 2)支持格林函 数方法、Berry 相位及微扰理论; 3)对 Monkhorst-Pack 特殊点的设定方便, 具有精确的泛函类型。VASP 可用于电子结构计算和量子力学-分子动力学模拟, 包括结构性质(键长、键角等晶格结构参数及能级、 能带等电子结构)、能量导数 (力常数和应力)、动力 学与弛豫 (从头算动力学、过渡态搜索)、磁性、对 静电场下的线性响应及多种光学性质等。利用该软件 框架大和泛函多的优点, 可对氧化铇的能量电子结构 执行精确计算, 如其光裂解水时的能量转变; 元素掺 杂下氧化物的电子结构及光学性质; 计算异质结材料 介电常数的实部与虚部、电荷转移、能量势垒等。

\section{3 其他软件程序}

1) WIEN 系列程序 ${ }^{[50]}$ 。WIEN 系列程序具有以 下特点: 基于 LINUX 平台, 稳定性高; 采用图形友 好交互界面, 操作难度低; 采用 FORTRAN 语言编 写，符合科研人员需求。

2) WinXMorph 程序 ${ }^{[51]}$ 。WinXMorph 是华盛顿 大学的免费程序, 通过输入晶体学数据, 可以创建 出逼真的静态或动态晶体形状(形貌)。

3) VESTA (Visualization for electronic and structural analysis)程序 ${ }^{[52]}$ 。VESTA 是一款免费的三维可 视化程序, 可给出结构模型及电子/核密度、晶体形 貌等结果。

另外还有 Band、Gaussian98 程序包、 PWSCF (Plane-wave self-consistent field)、Crystal 等程序 ${ }^{[50]}$, 这些程序均可单独(或与上述其他第一性原理计算 软件相结合)对纳米氧化铇的多项应用性能进行模 拟与计算。

\section{3 第一性原理计算在纳米氧化铇性能 研究中的应用}

具有多种功能的第一性原理计算软件在纳米氧 化铇的气敏性能、光催化性能、电致变色性能、电 极材料等领域都有应用, 在氧化铇生长机理等方面 的探索中也有涉及, 多种第一性原理计算软件(MS、 VASP、VESTE、WinXMorph 等)搭配不同的交换泛 函方法(GGA + PBE、PBE0、B3LYP、HSE06、DFT-D3 等), 可用于氧化铇的电子、光学、表面态、缺陷态 及掺杂等方面的研究。

\section{1 材料气敏研究}

人类在创造文明的同时也在不断地破坏环境, 
迅猛发展的现代工业所引发的生态问题不容乐观， 多种有毒、有害、可燃性气体排入大气，不仅造成 环境污染、破坏生态平衡，也在时刻危害着人类健 康，因此急需对这些有毒有害气体进行检测和控制, 相关研究已取得了一定进展 ${ }^{[53-55]}$ 。为了更深入地从 微观角度解释氧化铇对气体的敏感性机理, 研究者 利用第一性原理, 探究了 $\mathrm{O}_{3}{ }^{[56]} 、 \mathrm{CO}^{[51,56-58]}$ 、丙酩 ${ }^{[27]}$ 、 $\mathrm{HCHO}^{[59]} 、 \mathrm{NO}^{[60]} 、 \mathrm{NO}_{2}{ }^{[24,61-63]}$ 等典型气体在 $\mathrm{WO}_{3}$ 表面的吸附及其气敏检测问题。

氧化铇的不同表面及相应的表面能会直接影响 气体分子在其上的吸附, 有研究发现 $\mathrm{WO}_{3}(001)$ 表面 的能量最低 ${ }^{[64]}$, 因此气体易于吸附于此表面。Zhao 等 ${ }^{[57]}$ 研究了 $\mathrm{CO}$ 分子在 $\mathrm{h}-\mathrm{WO}_{3}(001)$ 表面 $\mathrm{O}$ 终端及 $\mathrm{WO}$ 终端的吸附特性, 发现 $\mathrm{CO}$ 分子在 $\mathrm{O}$ 终端发生 的是化学吸附, 因此在 $\mathrm{O}$ 终端的吸附活性比在 $\mathrm{WO}$ 终端的更强，吸附稳定性也更好。

Tang 等 ${ }^{[59]}$ 研究了 $\mathrm{HCHO}$ 分子在 $\mathrm{h}-\mathrm{WO}_{3}(001)$ 上 的吸附能、电子结构及电荷分布情况，对比了 $\mathrm{h}-\mathrm{WO}_{3}$ 不同位点的吸附性能。图 1(A)中给出了 $\mathrm{HCHO}$ 分子 在 $\mathrm{h}-\mathrm{WO}_{3}(001)$ 上 $\mathrm{W}_{5}$ 及 $\mathrm{O}_{7}$ 位点的吸附模型，图 1(B) 的电荷差分密度图直观地表现了这两个位点在吸附 前后的电荷密度变化。计算表明两位点在吸附 $\mathrm{HCHO}$ 后均出现电荷转移, 说明 $\mathrm{h}-\mathrm{WO}_{3}(001)$ 对 $\mathrm{HCHO}$ 具有 良好的吸附性，可用于检测 $\mathrm{HCHO}$ 分子。

气体在氧化铇表面的吸附能力及吸附位点还与 氧化铇存在的氧缺陷密切相关, 氧缺陷的存在会增 强氧化铇对气体的敏感性。韩啸等 ${ }^{[60]}$ 运用 MS 软件 的 CASTEP 模块, 采用搜索过渡态的方法研究了 $\mathrm{NO}_{2}$ 分子在具有氧空位的 $\mathrm{WO}_{3}(001)$ 表面的吸附过 程。计算表明, 在 $\mathrm{WO}_{3}(001)$ 的 $\mathrm{O}$ 终端表面, $\mathrm{NO}_{2}$ 易 于吸附在氧缺陷处的桥氧原子上; 在 WO 终端表面, $\mathrm{NO}_{2}$ 中的一个 $\mathrm{O}$ 原子会填充到氧空位处, 由此生成 $\mathrm{NO}$ 分子, 且所生成的 NO 会吸附到 WO 终端的 W 原子上。

秦玉香课题组 ${ }^{[24,61-62]}$ 揭示了 $\mathrm{NO}_{2}$ 气体在 $\mathrm{W}_{18} \mathrm{O}_{49}$ 纳米线上的吸附问题: 相较于 $\mathrm{WO}_{3}$, 含有氧缺陷的 $\mathrm{W}_{18} \mathrm{O}_{49}$ 纳米线在吸附 $\mathrm{NO}_{2}$ 分子过程中转移的电荷 更多，说明其对 $\mathrm{NO}_{2}$ 的吸附能力更强; 通过选取两 种简单而有代表性的 $\mathrm{W}_{18} \mathrm{O}_{49}$ 结构单元 $\mathrm{NW} 1$ 和 NW2(图 2 (A)), 采用以能量为导向的吸附位点选择 方法, 分别研究了两种模型上可能的吸附位点(图 2 (B))：对于 $\mathrm{NW} 1$ 模型, $\mathrm{NO}_{2}$ 分子倾向于吸附在 $\mathrm{O}_{2 c}$ 位点, $\mathrm{NO}_{2}$ 分子在 $\mathrm{NW} 2$ 模型中则易于吸附在 $\mathrm{W}_{6 \mathrm{c}}$ 位点。

第一性原理计算表明, 氧化铇表面的气体吸附 行为, 也会反过来对材料的氧缺陷及能级等产生影
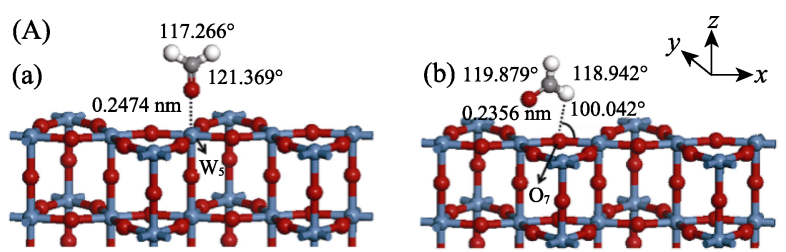

(B) (a) (b)

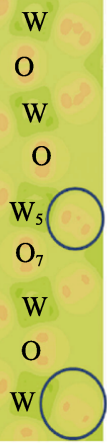

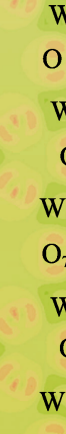

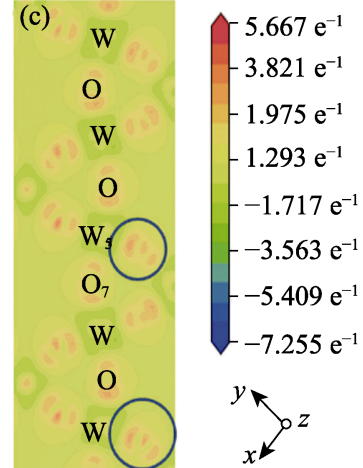

图 1 (A) $\mathrm{HCHO}$ (红球、白球、黑球分别代表 $\mathrm{O} 、 \mathrm{H}$ 和 $\mathrm{C}$ 原 子)在优化后的 $\mathrm{h}-\mathrm{WO}_{3}$ 晶体结构 (a) $\mathrm{W}_{5}$ 位点和(b) $\mathrm{O}_{7}$ 位点吸附; (B) $\mathrm{HCHO}$ 在 $\mathrm{h}-\mathrm{WO}_{3}$ (001)面(a)吸附前、(b)吸附于 $\mathrm{W}_{5}$ 位点和 (c)吸附于 $\mathrm{O}_{7}$ 位点的模型 ${ }^{[59]}$

Fig. 1 (A) Optimized adsorption structures of $\mathrm{HCHO}$ with red, white and black balls representing $\mathrm{O}, \mathrm{H}$ and $\mathrm{C}$, respectively, on $\mathrm{W}_{5}\left(\mathrm{HCHO}-\mathrm{W}_{5}\right.$ configuration) (a) and $\mathrm{O}_{7}\left(\mathrm{HCHO}-\mathrm{O}_{7}\right.$ configuration) (b) sites of WO-terminated $\mathrm{h}-\mathrm{WO}_{3}$ (001) surface; (B) Calculated electron density difference of the clean (001) surface (a), HCHO-absorbed on (001) surface for $\mathrm{HCHO}_{-} \mathrm{W}_{5}$ (b) and $\mathrm{HCHO}-\mathrm{O}_{7}$ (c) configurations ${ }^{[59]}$
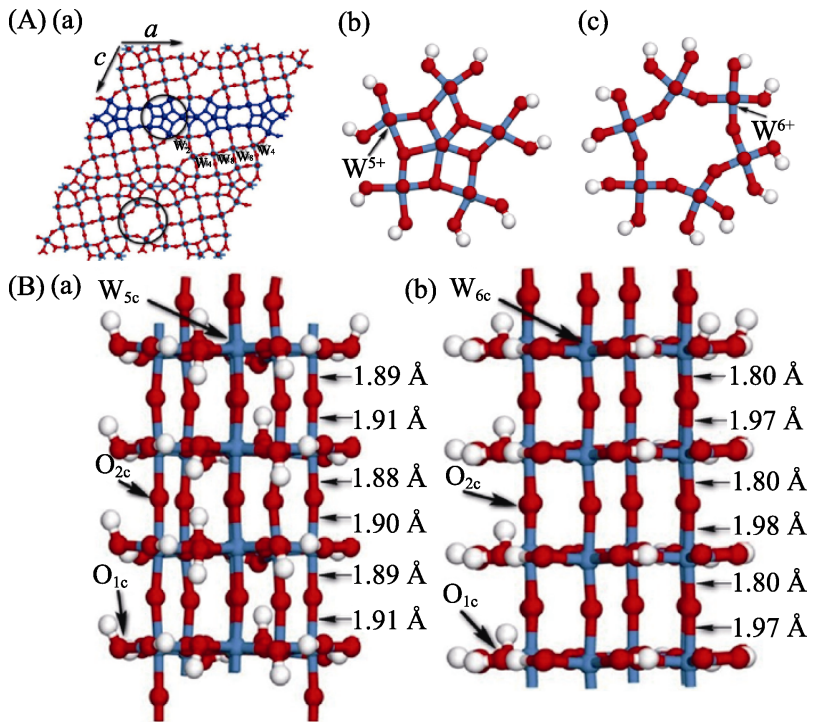

图 2 (A) $\mathrm{W}_{18} \mathrm{O}_{49}$ 纳米线超胞模型(a)及从中选出的结构单元 NW1(b)和 NW2(c)的俯视图, 其中 NW1 模型含有的 $\mathrm{W}^{5+}$ 更 多, $\mathrm{NW} 2$ 模型含有的 $\mathrm{W}^{6+}$ 更多; $(\mathrm{B}) \mathrm{W}_{18} \mathrm{O}_{49}(010)$ 纳米线优化后 的 NW1(a) 和 NW2(b)模型及 $\mathrm{NO}_{2}$ 可能的吸附位点 ${ }^{\text {[24, 61-62] }}$

Fig. 2 (A) Monoclinic structure (a) of $\mathrm{W}_{18} \mathrm{O}_{49}$ nanowires supercell model and its top views of NW1 (b) and NW2 (c), where NW1 and NW2 include largely cations $\mathrm{W}^{5+}$ and cations $\mathrm{W}^{6+}$, respectively; (B) Optimized models for NW1 (a) and $\mathrm{NW} 2$ (b), of $\mathrm{W}_{18} \mathrm{O}_{49}(010)$ nanowires ${ }^{[24,61-62]}$.

$\mathrm{O}, \mathrm{W}$ and $\mathrm{H}$ atoms are represented by red, blue and white balls, respectively $(1 \AA=0.1 \mathrm{~nm})$ 
响, 进一步改变材料的气敏性能。如 $\mathrm{O}_{3}$ 在 $\mathrm{WO}_{3}$ 薄膜 表面的气体吸附过程中被还原, 会减少氧化铇中的氧 缺陷数量(氧空位被消耗), 从而降低材料的电导性 ${ }^{[56]}$ 。

$\mathrm{WO}_{3}(001)$ 表面上 $\mathrm{CO}$ 的氧化则会诱导氧缺陷产 生, 增强材料的电导性。同时, 缺陷态又能再次被空 气中的 $\mathrm{O}_{2}$ 氧化后释放 $\mathrm{CO}_{2}$ 。随着 $\mathrm{CO}_{2}$ 的释放, 材料 又恢复成最初的 $\mathrm{WO}_{3}(001)$ 表面状态 ${ }^{[58]}{ }_{0} \mathrm{NO}_{2}$ 分子在 氧化铇表面发生吸附后, 氧化铇的所有能带均向低 能方向偏移, 且带隙中出现新能级, 使费米能级向 高能方向移动, 增强了氧化铇对 $\mathrm{NO}_{2}$ 的敏感性 ${ }^{[63]}$ 。

此外, 氧化铇通过掺杂而引入掺杂能级, 如在 氧化铇中掺入 $\mathrm{Ti}$ 或 $\mathrm{Sb}$ 杂质 ${ }^{[61,65]}$, 也可有效增强材 料的气敏性。主要是因为杂质能级可增大氧化铇的 禁带宽度, 使材料电阻增大, 导致气敏性得到增强。

\section{2 光催化应用}

目前全球经济的快速发展造成了环境恶化与能 源危机等问题, 引起了国际社会的普遍关注。因此 大力发展清洁能源, 以满足人类日益增长的能源需 求, 具有现实价值和重大意义。在解决环境恶化及 能源危机的各种方法中, 光催化技术因其绿色、环 保、无污染、应用领域(裂解水产氢、降解有机物、 有机合成)广泛等优点受到广泛重视。

光催化分解水产氢不仅可以获得清洁的氢气能 源, 还可以获得用以降解有机污染物的 $-\mathrm{OH} 、 \mathrm{O}_{2}-$ 等 活性基团, 有利于环境污染治理。晶格稳定性对于 光催化分解水十分重要, 一般来说, 晶格结构越稳 定, 越有利于产氢。使用第一性原理计算各种结构 $\mathrm{WO}_{3}$ 催化剂的晶格动力学参数发现 ${ }^{[66-67]}, \alpha-\mathrm{WO}_{3}$ 、 $\beta-\mathrm{WO}_{3} 、 \gamma-\mathrm{WO}_{3}$ 和 $\delta-\mathrm{WO}_{3}$ 的对称性扭曲可提高 $\mathrm{WO}_{6}$ 八面体晶格稳定性, 而 $\mathrm{O}-\mathrm{W}-\mathrm{O}$ 键的伸缩振动及轻 微的弯曲振动则造成晶体结构不稳定。在这些研究 案例中, 研究者发现, 既往使用第一性原理计算 $\mathrm{WO}_{3}$ 催化剂的晶格动力学参数出现的偏低问题, 可用杂化
泛函方法 PBE0 来计算能带结构和态密度加以矫正。

不可忽视的是, 空位及缺陷结构也对 $\mathrm{WO}_{3}$ 裂解 水的效率及光电性能产生重要影响(图 3(a)) ${ }^{[28]}$ 。无 氧缺陷的体 $\mathrm{WO}_{3}$ 中, 价带由 $\mathrm{O} 2 \mathrm{p}$ 轨道占据, 导带由 $\mathrm{W} 5 \mathrm{~d}$ 轨道占据; 图 3(b)中, 当向 $\mathrm{WO}_{3}$ 中引入一个氧 空位后, 材料的费米能级从价带转移至导带, 所形 成的带氧缺陷 $\mathrm{WO}_{3}$ 具有半金属性, 即发生了莫特转 变, 转变为 $\mathrm{n}$ 型半导体; 同时氧空位引起带隙窄化, 增强材料的电导及红外光响应性, 提升分解水效 率。引入氧缺陷还可使 $\mathrm{WO}_{3}$ 超薄纳米片由传统半导 体变成简并半导体 ${ }^{[68-69]}$, 导致更高的导电性和更为 合适的氢吸附自由能，从而提升其电解水产氢能力。

第一性原理计算可深入了解 $\mathrm{WO}_{3}$ 掺杂对其带 隙大小、带边位置、载流子密度等的影响。Valentin 课题组 ${ }^{[70]}$ 采用 B3LYP 杂化泛函研究了掺杂 $\mathrm{WO}_{3}$ 的 电子特性, 发现用 $\mathrm{S}$ 替代 $\mathrm{WO}_{3}$ 中的晶格 $\mathrm{O}$, 或用 $\mathrm{Mo} 、 \mathrm{Cr}$ 离子替代晶格 $\mathrm{W}$, 均可以窄化带隙, 从而提 升材料催化裂解水的性能; 用低价 $\mathrm{Ti} 、 \mathrm{Zr}$ 及 $\mathrm{Hf}$ 离 子替代 $\mathrm{W}$ 则会宽化带隙, 并使材料导带边移向能量 更高处, 且低价离子替代 $\mathrm{W}$ 后会诱导补偿缺陷的形 成, 有利于光催化产氢。Zhang 等 ${ }^{[71]}$ 借助 DFT 计算 证明了 $\mathrm{WO}_{3}$ 掺杂 $\mathrm{Fe}$ 后更易于形成氧缺陷, 增加载 流子密度, 提升分解水能力。

此外, 掺杂还对材料的光学性质产生重要影响, 使其在光催化应用方面具有优异表现。非金属 $\mathrm{S}$ 掺 杂及 $\mathrm{W}(\mathrm{O})$ 本征空位对 $\mathrm{WO}_{3}$ 光学表现影响显著 ${ }^{[23]}$ : 阴离子 $\mathrm{S}$ 掺杂使得光量子向低能量范围转换, 而阳 离子 $\mathrm{S}$ 掺杂使得 $\mathrm{WO}_{3}$ 的有效能带变窄, 更有利于产 生光生电子一空穴对, 成为有效光电流转换的关键 因素; 而 $\mathrm{W}(\mathrm{O})$ 本征空位会使光的吸收边发生红移, 提高光的利用率。利用第一性原理还可以计算材料 介电常数的实部和虚部, 得到材料的光吸收谱, 从 光响应范围及载流子寿命等本质特征上探讨掺杂
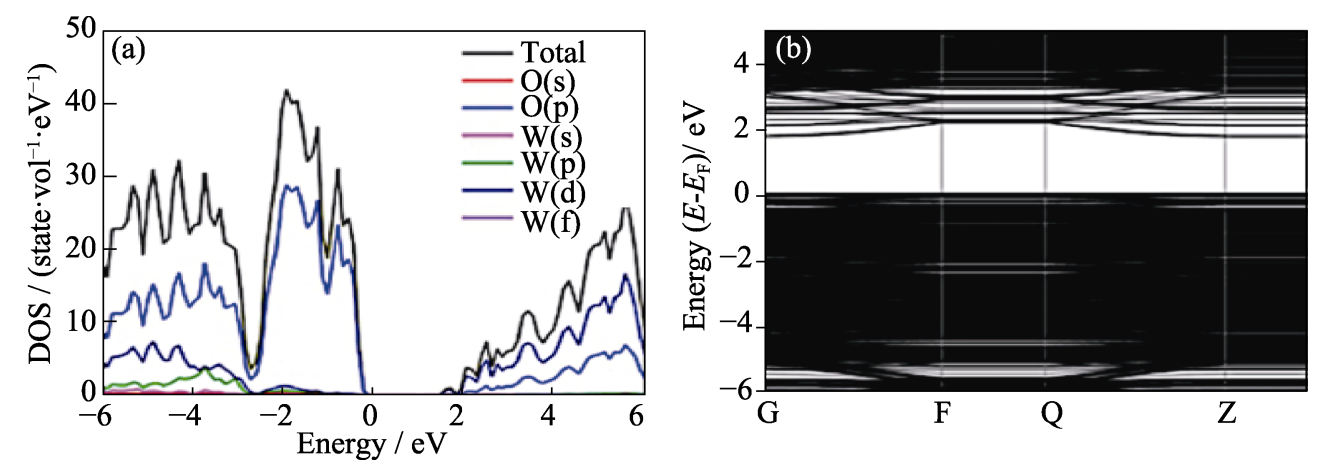

图 3 (a)无氧空位的 $\mathrm{WO}_{3}$ 体材料的态密度(DOS)及态密度投影(PDOS)图和(b)具有一个氧空位 $\mathrm{WO}_{3}(002)$ 面的带结构 ${ }^{[28]}$

Fig. 3 (a) Density of states and projected density of states of bulk $\mathrm{WO}_{3}$ without oxygen vacancy, and (b) structure of $\mathrm{WO}_{3}(002)$ with one oxygen vacancy ${ }^{[28]}$

Colorful images showing on website 
材料催化性能的优劣 ${ }^{[25]}$ 。

缺陷结构也会使材料内部产生自掺杂现象。借 助 MS 软件的 CASTEP 模块研究发现, $\mathrm{WO}_{3-x} / \mathrm{TiO}_{2-x}$ 纳米管材料(图 4(a))具有较高的光电转换效率及较 强的光电流响应性的原因, 就在于缺陷结构引入的 氧空位使得氧化钣中形成了 $\mathrm{Ti}^{3+}$ 自掺杂(图 4(b)), 并 使得氧化铇由半导体性转变为金属性, 产生了局域 表面等离子激元效应。在 $\mathrm{Ti}^{3+}$ 自掺杂及局域表面等 离子激元效应的协同作用下，电荷迁移效率得到明 显提升，由此极大地提高了材料光催化降解污染物 的活性 ${ }^{[72]}$ 。

在光催化有机合成方面，有研究者将多种表征 手段和第一性原理计算相结合，模拟分析了缺陷型 氧化铇表面吸附苄胺分子的能力及有氧氧化偶联反 应，发现氧空位作为不饱和位点，易于吸附并激发 氧分子，产生超氧基团，促进有机合成反应进行，从 微观层面解释了缺陷可提升氧化铇在有机合成反应 中催化性能的机制 ${ }^{[73-76]}$ 。

\section{3 电致变色应用}

随着人们对节能环保材料的日益关注，能够节 约能源、对太阳能高效利用的智能窗进入人们的视 野, 智能窗可动态调节光强、热辐射量及散热量 ${ }^{[77-78]}$, 满足人们对室内供暖、制冷和隔热的不同需求。作 为性能优越的电致变色材料, 氧化铇在智能窗领域 的应用前景广阔，越来越受到人们的关注。

近些年来, 为完善和提高氧化铇的电致变色性 能, 科研人员采用多种途径对其进行模拟计算。最 初认为立方结构的氧化铇结构简单、对称性好、操

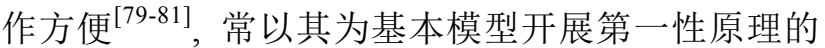
模拟计算工作。但因为模型被过度简化, 计算结果

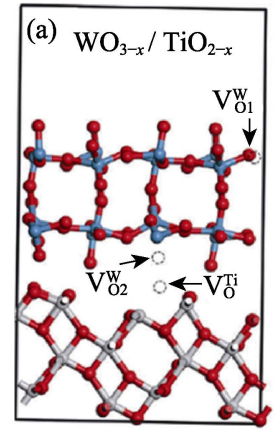

(b)

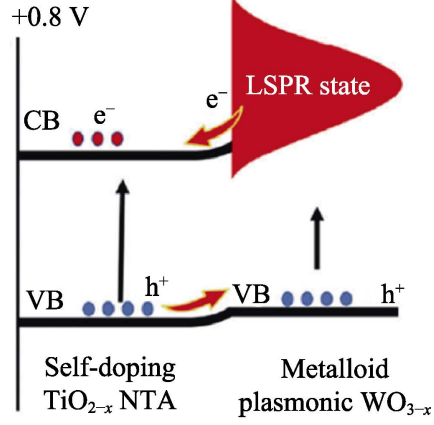

图 4 (a) $\mathrm{WO}_{3-x} / \mathrm{TiO}_{2-x}$ 的几何优化平衡结构(红球、蓝球、白 球分别代表 $\mathrm{O} 、 \mathrm{~W}$ 和 $\mathrm{Ti}$ 原子), 及(b) $\mathrm{WO}_{3-x} / \mathrm{TiO}_{2-x}$ 中的 $\mathrm{Ti}^{3+}$ 自掺杂, 表面等离子激元效应(LSPR)及电荷迁移示意图 ${ }^{[72]}$

Fig. 4 (a) Geometrically optimized equilibrium configuration of $\mathrm{WO}_{3-x} / \mathrm{TiO}_{2-x}$ with red, blue and white balls representing $\mathrm{O}$, $\mathrm{W}$ and $\mathrm{Ti}$, respectively, and (b) schematic diagram of the self-doping $\mathrm{Ti}^{3+}$, localized surface plasmon resonance (LSPR), and charge transfer in $\mathrm{WO}_{3-x} / \mathrm{TiO}_{2-x}{ }^{[72]}$
经常与实际数值有较大出入。随后人们开始重视氧 化铇中被忽略的铇氧八面体倾斜畸变和结构畸变, 并在此基础上开展了氧化铇材料对光的吸收、辐射、 能量转化等的研究工作。

秦京运等 ${ }^{[29]}$ 采用第一性原理计算的方法, 研究 了 $\mathrm{Ti}$ 掺杂 $\mathrm{h}-\mathrm{WO}_{3}$ 的电致变色性能。结果表明 $\mathrm{Ti}$ 掺 杂后 $\mathrm{h}-\mathrm{WO}_{3}$ 的晶格体积增大(图 5)、系统能量显著 降低，体系的稳定性得到明显提升，且掺杂后价带 整体下移，形成简并态，晶体从半导体向导体转变， 但体系仍保持 $\mathrm{n}$ 型电导率，一系列电子结构的变化, 最终导致 $\mathrm{Ti}$ 掺杂 $\mathrm{h}-\mathrm{WO}_{3}$ 对近红外光的吸收性增强, 具有近红外光辐射屏蔽性能。

$\mathrm{Yang}^{[82]}$ 通过 VASP 软件, 对 $\mathrm{WO}_{x}-\mathrm{WSe}_{2}-\mathrm{MoS}_{2}$ p-n 结型复合单片材料进行了带对齐分析及第一性 原理计算，计算中采用了基于 GGA 的 DFT-D3 色散 矫正交换泛函。在这种复合材料中，层与层间的 $\mathrm{WSe}_{2}$ 可以直接氧化成原子尺度的 $\mathrm{WO}_{x}$ 薄层, 该薄 层可以作为提升空穴抽取速率的电荷传输层(图 6), 极大增强了 $\mathrm{WSe}_{2}-\mathrm{MoS}_{2} \mathrm{p}-\mathrm{n}$ 结型光电设备的光响应 性，有效提升了设备的能量转化效率。

考虑到当前社会对智能窗材料的迫切需求，从 第一性原理的角度, 对氧化铇材料在电致变色应用 中的研究给予理论指导与计算辅助, 显得尤为重 要。但应该注意到, 目前的理论计算工作在电致变 色应用中仍存在诸多与实验不符的现象或难以解决 的问题, 如理论计算中轨道的选取及键长键角的些 微改变, 均会影响计算与实验结果之间的误差 ${ }^{[83]}$ 。 因此, 仍需研究者们在这一应用领域进一步探索。

\section{4 电池电极材料}

随着能源技术的不断发展，锂离子电池为人们 的生产和生活带来了极大的便利, 因此研究其组成 结构及应用性能尤为必要。采用第一性原理可以方 便直观地给出示意模型，说明材料的吸附、扩散等 问题，更便捷地对复杂的锂嵌入脱出理论进行剖析，

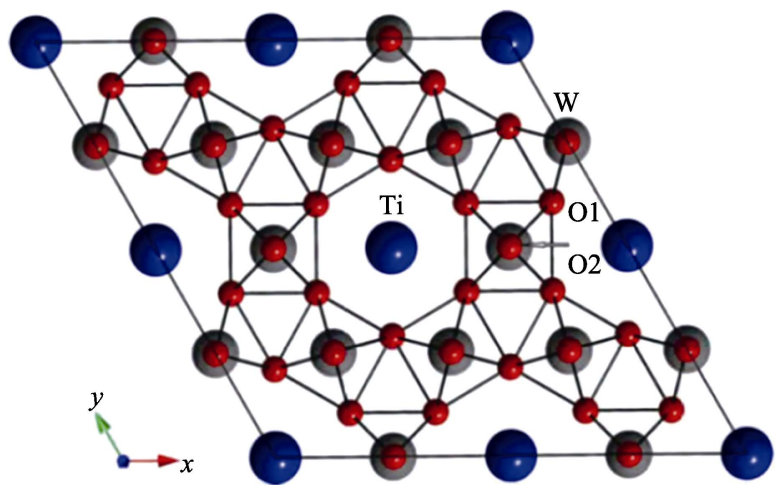

图 $5 \mathrm{Ti}$ 掺杂 $\mathrm{h}-\mathrm{WO}_{3}$ 的超晶胞俯视图 ${ }^{[29]}$

Fig. 5 Top view of the supercell of Ti-doped h- $\mathrm{WO}_{3}{ }^{[29]}$ 

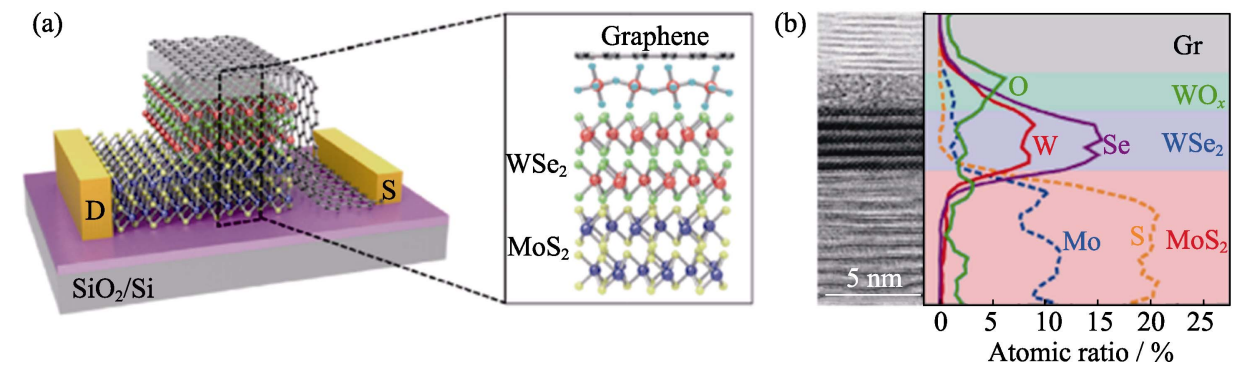

图 $6 \mathrm{WSe}_{2}-\mathrm{MoS}_{2} \mathrm{p}-\mathrm{n}$ 异质结(a)有氧化层的 $\mathrm{WO}_{x} / \mathrm{WSe}_{2} / \mathrm{MoS}_{2}$ 设备截面示意图(粉球、蓝球、绿球、紫球、 黄秋分别代表 W、O、Se、Mo 和 S 原子)和(b)截面的 HR-TEM 照片及 EDS 图 ${ }^{[82]}$

Fig. 6 Monolithically band-engineered $\mathrm{WSe}_{2}-\mathrm{MoS}_{2}$ p-n heterojunction ${ }^{[82]}$

(a) Schematic illustration of the vertical $\mathrm{WSe}_{2}-\mathrm{MoS}_{2}$ device with the $\mathrm{WO}_{x}$ layer, where pink, blue, green, purple and yellow balls represent $\mathrm{W}, \mathrm{O}$, Se,

Mo and S, respectively; (b) Cross-sectional HR-TEM image and EDS elemental line profiles across the $\mathrm{WO}_{x} / \mathrm{WSe}_{2} / \mathrm{MoS}_{2}$ heterointerfaces

有益于氧化铇作为电池电极材料的应用研究。

运用 MS 软件构建模型-VESTA 软件优化可视 化效果-VASP 软件开展计算的方法 ${ }^{[84]}$, 可以深入研 究 $\mathrm{WO}_{3}$ 作为锂离子电池负极材料的性能: $\mathrm{WO}_{3}$ 体系 具有三个稳定的吸附锂位置, 且理论最大吸附数量 为 6 ; 利用集团展开法等对 $\mathrm{WO}_{3}$ 的扩散机制进行分 析, 发现锂离子更易于沿 $\mathrm{WO}_{3}$ 中路径较短的氧六边 形中 $\mathrm{O}-\mathrm{O}$ 键对应的方向进行扩散。

Wadsley-Roth 晶体剪切相形成的化合物作为锂 离子电池电极具有优异性能, 但该类材料往往具有 复杂的晶体结构, 难以对其内部电子结构及动力学 性质进行研究。近来人们开始借助第一性原理计算 辅助开展此类研究。如 Kocer 等 ${ }^{[85}$ 采用第一性原理 计算及枚举法对三种不同的铌铇氧化物, $\mathrm{Nb}_{12} \mathrm{WO}_{33}$ 、 $\mathrm{Nb}_{14} \mathrm{~W}_{3} \mathrm{O}_{44} 、 \mathrm{Nb}_{16} \mathrm{~W}_{5} \mathrm{O}_{55}$ 进行了研究(图 7), 揭示了长 程结构与局部结构变化的相关性: 在锂嵌入过程中, 局部结构畸变的消失引起固定晶向晶胞的收缩, 对 材料的体积膨胀起到缓冲作用。由于块材的密闭性, (a)

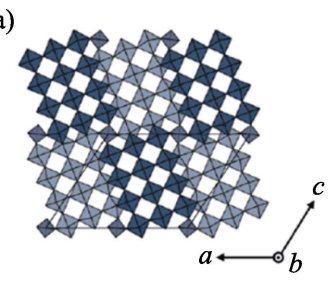

$\mathrm{Nb}_{12} \mathrm{WO}_{33}$

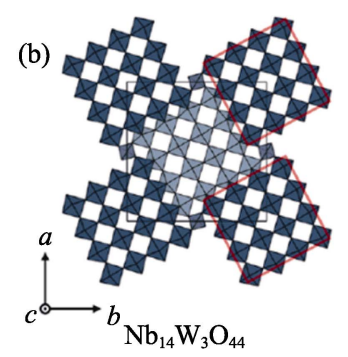

(c)

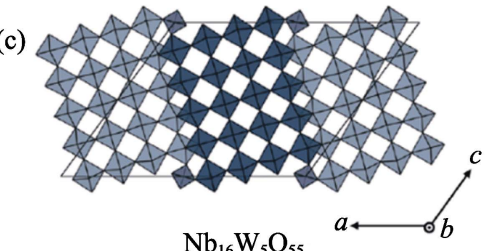

图 7 Wadsley-Roth 相晶体结构 ${ }^{[85]}$

Fig. 7 Crystal structures of Wadsley-Roth phases ${ }^{[85]}$

(a) $\mathrm{Nb}_{12} \mathrm{WO}_{33}$ (space group C2); (b) $\mathrm{Nb}_{14} \mathrm{~W}_{3} \mathrm{O}_{44}$ (I4/m); (c) $\mathrm{Nb}_{16} \mathrm{~W}_{5} \mathrm{O}_{55}$ (C2)
在最初锂嵌入时, 铌铇氧化物剪切结构只具有少量 的局部电子, 但是继续锂化会使其迅速出现金属 性。作者提出的机械原理源于晶体剪切结构, 与组 成无关, 有望应用于铌钛氧化物及纯铌氧化物晶体 的剪切相。

第一性原理还可以对甲醇燃料电池阴极上的气 体吸附行为进行微观分析 ${ }^{[86]}$ 。 $\mathrm{CH}_{4}$ 气体在 $\mathrm{W}_{18} \mathrm{O}_{49}$ 纳米线上的吸附能最高, $\mathrm{H}_{2} \mathrm{O}_{2} 、 \mathrm{CO} 、 \mathrm{O}_{2}$ 分子次之; 且 气体吸附后, 电池阴极反应的吸附能为负值, 即吸 附反应为放热反应, 说明该电池阴极上的吸附稳定 性良好。此外, 研究人员还利用 CASTEP 给出了一 种新的表面吸附模型— $\mathrm{NW}-\mathrm{Co}(\mathrm{Co})$ 原子吸附在 $\mathrm{W}_{18} \mathrm{O}_{49}$ 纳米线表面的 $\mathrm{W}$ 原子处(图 8) ${ }^{[86]}$, 研究了气 体分子 $\left(\mathrm{CO} 、 \mathrm{CH}_{4} 、 \mathrm{O}_{2} 、 \mathrm{H}_{2} \mathrm{O}_{2}\right)$ 在 NW-Co 上的吸附效 果。NW-Co 具有分裂 $\mathrm{O}_{2}$ 及 $\mathrm{H}_{2} \mathrm{O}_{2}$ 中 $\mathrm{O}-\mathrm{O}$ 键的能力, 弥补了上述 $\mathrm{W}_{18} \mathrm{O}_{49}$ 纳米线材料对 $\mathrm{O}_{2}$ 及 $\mathrm{H}_{2} \mathrm{O}_{2}$ 吸附性 不足的缺点, 因此将其作为燃料电池阴极的催化剂 载体具有较好的应用前景。

\section{5 其他方面应用}

第一性原理除了应用于氧化铇的气敏、光催化、 电致变色、电池电极材料等功能性领域, 还应用于 氧化铇的生长机制、生化应用、稳定性求解、制备 超细铇粉等方面的研究。

一维材料的生长机理问题一直是纳米材料领域 的关注热点。张正飞等 ${ }^{[87-89]}$ 研究了 $\mathrm{W}_{18} \mathrm{O}_{49}$ 纳米线和 纳米管在没有催化剂时的生长机理。作者首先使用 WinXMorph 程序预测了 $\mathrm{W}_{18} \mathrm{O}_{49}$ 的晶体形状, 然后 以 VASP 软件构建了 $\mathrm{W}_{18} \mathrm{O}_{49}$ 平板模型, 发现在适量 $\mathrm{O}_{2}$ 气氛下 $\mathrm{W}_{18} \mathrm{O}_{49}(010)$ 面的能量最高, 从而证明 $\mathrm{W}_{18} \mathrm{O}_{49}$ 纳米线的择优生长方向为 $[010]$ 。Chen 等 ${ }^{[00]}$ 以分子动力学(Molecular dynamic, MD) 模型研究了 $\mathrm{h}-\mathrm{WO}_{3}$ 纳米棒生长过程中, 阳离子 $\mathrm{NH}_{4}{ }^{+}$嵌入的影 响。交互能计算结果表明, 嵌入适当浓度的阳离子 可以诱导晶体沿(001)方向加速生长, 形成一维结构; 
(a)

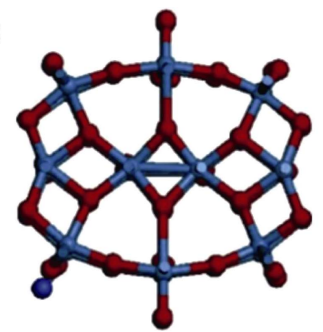

(c)

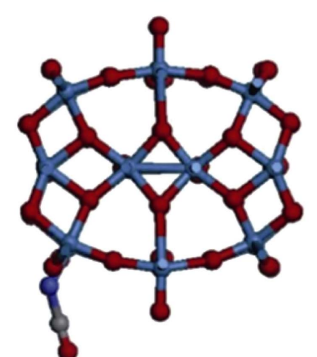

(e)

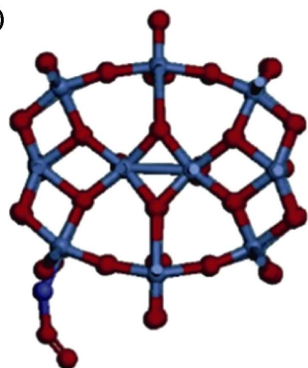

(b)

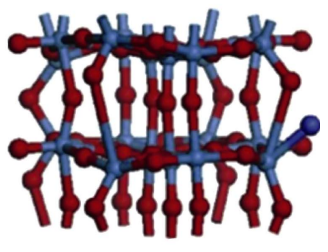

(d)

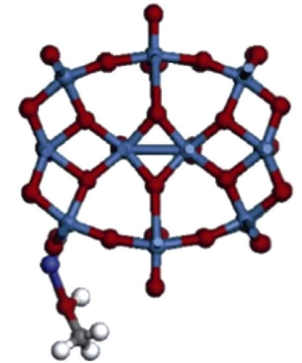

(f)

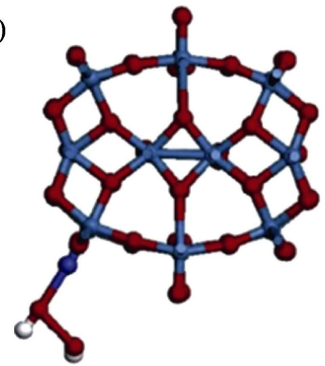

图 8 优化后的不同气体分子在 $\mathrm{W}_{18} \mathrm{O}_{49}$ 上的吸附模型(蓝 球、紫球、红球、灰球、白球分别代表 $\mathrm{W} 、 \mathrm{Co} 、 \mathrm{O} 、 \mathrm{C}$ 和 $\mathrm{H}$ 原子 $)^{[86]}$

Fig. 8 Optimized adsorption model of different gas molecules on $\mathrm{W}_{18} \mathrm{O}_{49}$ with blue, purple, red, gray and white balls representing $\mathrm{W}, \mathrm{Co}, \mathrm{O}, \mathrm{C}$ and $\mathrm{H}$, respectively ${ }^{[86]}$

(a, b) Adsorbed cobalt atom on the tungsten atom of NW (NW-Co); (c) Carbon monoxide molecule adsorbed on the NW-Co; (d) Methanol molecule adsorbed on the NW-Co; (e) Oxygen molecule adsorbed on the NW-Co; (f) Hydrogen peroxide molecule adsorbed on the NW-Co

$\mathrm{NH}_{4}{ }^{+}$离子处于氧化钨六方通道中时(图 9), 体系结 构最稳定，因为 $\mathrm{NH}_{4}{ }^{+}$的离子半径大，与晶胞的相互 作用强。此模拟计算结果的可靠性得到了实验证实。

也有学者采用 MS 的 Forcite+模块, 以 MD 模型 模拟研究了氧化铇的生物化学性能。如 Jiang 等 ${ }^{[91]}$ 以 $\mathrm{MD}$ 模型研究了手性陶瓷 $\mathrm{WO}_{3-x} \cdot \mathrm{H}_{2} \mathrm{O}$ 纳米颗粒基 于对称光学的性能, 验证了对称性从氨基酸转移到 $\mathrm{WO}_{3-x}$ 晶胞的事实, 证明了 $\mathrm{MD}$ 模型在手性光学及 生物化学方面的应用价值。

此外, 为了研究氧化钨作为表面增强拉曼散射 材料的应用性能，顾凌军等 ${ }^{[92]}$ 采用 VASP 软件对含 氧缺陷的 $\mathrm{W}_{18} \mathrm{O}_{49}$ 开展研究, 预测了载流子掺杂对 $\mathrm{W}_{18} \mathrm{O}_{49}$ 能带结构的调控作用, 发现氧空位会在 $\mathrm{W}_{18} \mathrm{O}_{49}$ 的禁带中引入杂质能级, 增加半导体的电子跃迁几 率。同时对比研究发现, 使用 HSE06 杂化泛函得到 的结果能够更好地与实验数据相符。其他学者也

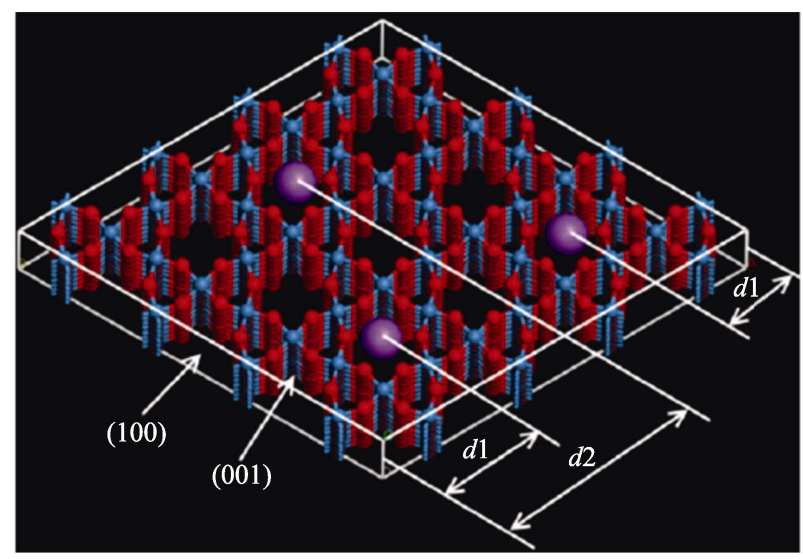

图 $9 \mathrm{~h}-\mathrm{WO}_{3}(100)$ 面不同的嵌入位点示意图(蓝球、红球、紫 球分别代表 $\mathrm{W} 、 \mathrm{O}$ 原子和阳离子 $)^{[90]}$

Fig. 9 Various intercalating sites corresponding to different distances to the $\mathrm{h}-\mathrm{WO}_{3}(100)$ surface with blue, red and purple balls representing $\mathrm{W}, \mathrm{O}$ and cations, respectively ${ }^{[90]}$

证实了这种做法在单斜、三斜以及立方晶系的 $\mathrm{WO}_{3}$ 中的适用性 ${ }^{[93-94]}$ 。

在氧化铇团簇的稳定性求解过程中, Sai 等 ${ }^{\left[{ }^{[95}\right]}$ 将 遗传算法与第一性原理计算相结合, 寻求最稳定的 $\left(\mathrm{WO}_{3}\right)_{n}$ 簇 $(n=2 \sim 12)$ 结构 (图 10), 并以 $\mathrm{MS}$ 的 $\mathrm{Dmol}^{3}$

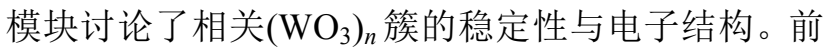
线轨道及电子态密度分析表明, 氧化铇中的价带由 $\mathrm{O} 2 \mathrm{p}$ 电子占据, 导带由 $\mathrm{W} 5 \mathrm{~d}$ 电子占据; 小 $\left(\mathrm{WO}_{3}\right)_{n}$ 簇 $(n=3,4)$ 为 $\mathrm{W}-\mathrm{O}$ 交替排布的环状结构, 从 $\left(\mathrm{WO}_{3}\right)_{8}$ 开 始则变为对称球状笼形结构。Wang 课题组 ${ }^{\left[{ }^{[96]}\right.}$ 也研 究了过氧化铇纳米簇的电子结构特性, 其研究主要 针对 $\mathrm{W}_{3} \mathrm{O}_{n}^{-}$及 $\mathrm{W}_{3} \mathrm{O}_{n}(n=7 \sim 10)$ 纳米簇体系, 研究发 现在 $n=7 \sim 10$ 的氧化铇中, 除 $\mathrm{W}_{3} \mathrm{O}_{10}{ }^{-}$中只有 $\mathrm{O} 2 \mathrm{p}$ 轨道 分离外, 其他氧化铇的 $\mathrm{O} 2 \mathrm{p}$ 和 W5d 都具有分离特征。

氧化铇也可以作为原料制备铇粉。氢气还原氧 化铇制备超细纳米铇粉是目前使用最广泛的铇粉制

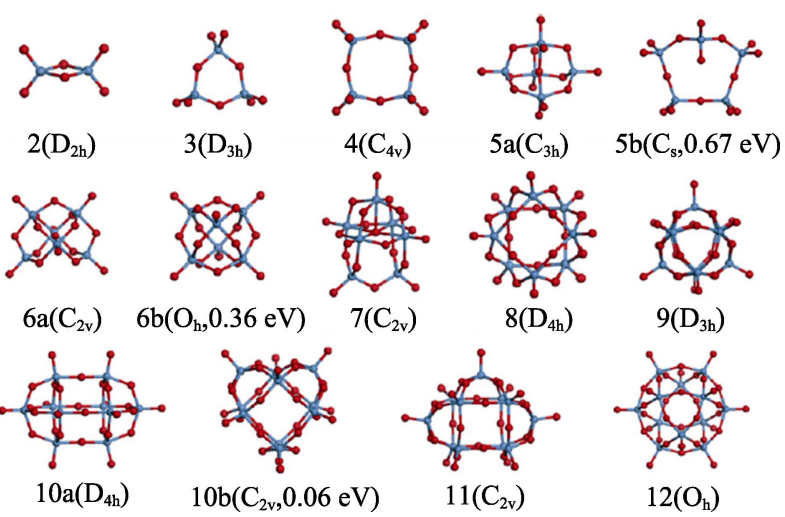

图 $10 \quad\left(\mathrm{WO}_{3}\right)_{n}$ 簇 $(n=2 \sim 12)$ 最低能量结构及其亚稳态同分异构 体 $(5 \mathrm{~b} 、 6 \mathrm{~b} 、 10 \mathrm{~b})$, 其中蓝球、红球分别代表 $\mathrm{W}$ 和 $\mathrm{O}$ 原子 ${ }^{[95]}$ Fig. 10 Lowest-energy structures of $\left(\mathrm{WO}_{3}\right)_{n}$ clusters $(n=2-12)$ and several metastable isomers (labeled as $5 \mathrm{~b}, 6 \mathrm{~b}, 10 \mathrm{~b}$ ) with blue and red balls representing $\mathrm{W}$ and $\mathrm{O}$, respectively ${ }^{[95]}$ 
备方法之一 ${ }^{[97]}$, 具有清洁、保质等优点, 目前已被 大规模应用于工业生产中。姜平国等 ${ }^{[98-101]}$ 以 MS 软 件的 CASTEP 模块研究了黄铇 $\left(\mathrm{WO}_{3}\right)$ 、紫铇 $\left(\mathrm{W}_{18} \mathrm{O}_{49}\right)$ 和蓝铇 $\left(\mathrm{W}_{20} \mathrm{O}_{58}\right)$ 的晶体结构, 以及氢气在这些氧化 铇晶体表面的吸附行为, 发现 $\mathrm{H}_{2}$ 分子与 $\mathrm{WO}_{3}(001)$ 面的原子距离大于 $0.08 \mathrm{~nm}$ 时, 作用较小, $\mathrm{H}_{2}$ 难以分 解，只能发生物理吸附; 小于 $0.08 \mathrm{~nm}$ 时, $\mathrm{H}_{2}$ 分解为 两个氢原子, 并与氧化铇表面原子发生化学反应, 生成 $\mathrm{W}-\mathrm{H}$ 键或 $\mathrm{H}-\mathrm{O}$ 键，属于化学吸附。作者前期 研究还表明, $\mathrm{WO}_{3} 、 \mathrm{~W}_{20} \mathrm{O}_{58}$ 和 $\mathrm{W}_{18} \mathrm{O}_{49}$ 都具有较强的 电子离域性, 通过改变其 $\mathrm{W}-\mathrm{O}$ 键长和键角能达到 表面弛豫的目的。

\section{4 结论与展望}

随着计算机硬件设备性能的提高和理论计算方 法的发展, 以及软件开发者的不解努力, 基于密度 泛函的第一性原理计算方法已逐渐渗透到物理、化 学、生物及工程等多个领域, 如本文所述, 第一性原 理在纳米氧化铇材料中的应用也已涉及气敏、光催 化、电致变色、锂电池材料及生长机制等多个研究 领域。但也应注意到, 目前的理论模拟计算还存在 一些问题, 需要后续研究加以解决:

1) 各种模型建立之初需要大量优化、重复、对 比、计算工作，一般超胞/非周期结构的计算时间较 长, 在进行专门计算时往往需借助超算等大型计算 设备来缩短时间与人力。

2) 模型构建过程中参数的选取常需要研究者 的经验判断, 处理方式复杂, 如交换关联泛函的选 择上，LDA、GGA 及其他杂化泛函方法各有利弊， 因此后续研究在选择参数时需要结合已有经验及前 人研究结果, 需要综合考虑精确度和计算量等进行 合理选取。

3) 目前研究者所构建的模型多是单分子模型, 研究分析的问题也以表面态居多，而对多分子交互 影响及材料整体结构变化的研究较少。实际应用中 的材料性能会受多种因素共同影响。因此研究者应 多注意理论计算结果与实际数据的对比分析与关联, 建立合理的研究模型。同时，可将第一性原理计算 与 $\mathrm{X}$ 射线近带吸收谱等先进表征方法相结合, 进行 更深层次的微观结构分析。

4) 鉴于近年来缺陷型氧化铇所表现出的优异 性能, 在今后研究中可以适当地加大对材料结构中 的畸变/空位等缺陷的关注, 为实际应用提供可靠依 据。研究者也应对比不同软件的特点, 合理采用一 种或多种模拟方法开展研究。值得一提的是, 一个 具有复杂算法的源代码，除开发者外几乎再无他人
理解应用, 因此研究者还应处理好使用计算软件时 可能出现的“不确定性”系统错误。

综上所述，基于第一性原理的模拟计算方法的 发展, 弥补了纯理论推导和纯实验研究的不足, 为 物理、化工及材料等领域提供了新颖有效的研究手 段。第一性原理在纳米氧化铇相关研究中的应用虽 尚处于起步阶段，且目前还有许多不足有待改进， 但其应用效果已得到广泛认可，相信理论模拟计算 与实验研究在未来将有更紧密的结合, 为氧化铇相 关性能的研究提供可靠、有效的辅助手段。

\section{参考文献:}

[1] YIN X T, LV P, LI J, et al. Nanostructured tungsten trioxide prepared at various growth temperatures for sensing applications. Journal of Alloys and Compounds, 2020, 825: 154105.

[2] NANDIYANTO A B D, OKTIANI R, RAGADHITA R, et al. Amorphous content on the photocatalytic performance of micrometer-sized tungsten trioxide particles. Arabian Journal of Chemistry, 2020, 13(1): 2912-2924.

[3] HE X, WANG X Y, SUN B N, et al. Synthesis of threedimensional hierarchical furball-like tungsten trioxide microspheres for high performance supercapacitor electrodes. RSC Advances, 2020, 10(23): 13437-13441.

[4] HAI G J, HUANG J F, CAO L Y, et al. Influence of oxygen deficiency on the synthesis of tungsten oxide and the photocatalytic activity for the removal of organic dye. Journal of Alloys and Compounds, 2017, 690: 239-248.

[5] LIU X F, ZHOU H, PEI S Z, et al. Oxygen-deficient $\mathrm{WO}_{3-x}$ nanoplate array film photoanode for efficient photoelectrocatalytic water decontamination. Chemical Engineering Journal, 2020, 381: 122740 .

[6] 赵林艳, 席晓丽, 桥佑书, 等. 纳米氧化钨的水热/溶剂热法制 备及应用的综述. 材料导报, 2019, 33(19): 3203-3209.

[7] QUAN H Q, GAO Y F, WANG W Z. Tungsten oxide-based visible light-driven photocatalysts: crystal and electronic structures and strategies for photocatalytic efficiency enhancement. Inorganic Chemistry Frontiers, 2020, 7(4): 817-838.

[8] PERSSON K. Materials project. https://materialsproject.org/.

[9] DEB S K. Opportunities and challenges in science and technology of $\mathrm{WO}_{3}$ for electrochromic and related applications. Solar Energy Materials and Solar Cells, 2008, 92(2): 245-258.

[10] DING Y, YANG I S, LI Z Q, et al. Nanoporous $\mathrm{TiO}_{2}$ spheres with tailored textural properties: controllable synthesis, formation mechanism, and photochemical applications. Progress in Materials Science, 2020, 109: 100620 .

[11] DONG P Y, HOU G H, XI X U, et al. $\mathrm{WO}_{3}$-based photocatalysts: morphology control, activity enhancement and multifunctional applications. Environmental Science-Nano, 2017, 4(3): 539-557.

[12] HAN L F, CHEN J L, ZHANG Y H, et al. Facile synthesis of hierarchical carpet-like $\mathrm{WO}_{3}$ microflowers for high $\mathrm{NO}_{2}$ gas sensing performance. Materials Letters, 2018, 210: 8-11.

[13] LI Y S, TANG Z L, ZHANG J Y, et al. Fabrication of vertical orthorhombic/hexagonal tungsten oxide phase junction with high photocatalytic performance. Applied Catalysis B-Environmental, 2017, 207: 207-217.

[14] HUNGE Y M, YADAV A A, MAHADIK M A, et al. A highly efficient visible-light responsive sprayed $\mathrm{WO}_{3} / \mathrm{FTO}$ photoanode for photoelectrocatalytic degradation of brilliant blue. Journal of the Taiwan Institute of Chemical Engineers, 2018, 85: 273-281.

[15] KARADENIZ S M, TATAR D, ERTUGRUL M, et al. Structural, 
optical and electrochromic properties of $\mathrm{WO}_{3}$ thin films prepared by chemical spray pyrolysis versus spin coating technique. Spectroscopy and Spectral Analysis, 2018, 38(9): 2982-2988.

[16] INAMDAR A I, CHAVAN H S, AHMED A A, et al. Nanograin tungsten oxide with excess oxygen as a highly reversible anode material for high-performance Li-ion batteries. Materials Letters, 2018, 215: 233-237.

[17] SHENG J P, ZHANG L, DENG L, et al. Fabrication of dopamine enveloped $\mathrm{WO}_{3-x}$ quantum dots as single-NIR laser activated photonic nanodrug for synergistic photothermal/photodynamic therapy against cancer. Chemical Engineering Journal, 2020, 383: 123071.

[18] ZHAO L Y, XI X L, LIU Y S, et al. Growth mechanism and visible-light-driven photocatalysis of organic solvent dependent $\mathrm{WO}_{3}$ and nonstoichiometric $\mathrm{WO}_{3-x}$ nanostructures. Journal of the Taiwan Institute of Chemical Engineers, 2020, 115: 339-347.

[19] ZHAO L Y, XI X L, LIU Y S, et al. Facile synthesis of $\mathrm{WO}_{3}$ micro/nanostructures by paper-assisted calcination for visiblelight-driven photocatalysis. Chemical Physics, 2020, 528: 110515.

[20] FAN Y S, XI X L, LIU Y S, et al. Growth mechanism of immobilized $\mathrm{WO}_{3}$ nanostructures in different solvents and their visible-light photocatalytic performance. Journal of Physics and Chemistry of Solids, 2020, 140: 109380.

[21] MANTHIRAM K, ALIVISATOS A P. Tunable localized surface plasmon resonances in tungsten oxide nanocrystals. Journal of the American Chemical Society, 2012, 134(9): 3995-3998.

[22] KIMURA Y, IBANO K, UEHATA K, et al. Improved hydrogen gas sensing performance of $\mathrm{WO}_{3}$ films with fibrous nanostructured surface. Applied Surface Science, 2020, 532: 147274.

[23] LI D, HUANG W Q, XIE Z, et al. Mechanism of enhanced photocatalytic activities on tungsten trioxide doped with sulfur: dopant-type effects. Modern Physics Letters B, 2016, 30(27): 1650340.

[24] QIN Y X, LIU M, YE Z H. A DFT study on $\mathrm{WO}_{3}$ nanowires with different orientations for $\mathrm{NO}_{2}$ sensing application. Journal of Molecular Structure, 2014, 1076: 546-553.

[25] CHEN Z J, CAO J X, YANG L W, et al. The unique photocatalysis properties of a $2 \mathrm{D}$ vertical $\mathrm{MoO}_{2} / \mathrm{WO}_{2}$ heterostructure: a firstprinciples study. Journal of Physics D-Applied Physics, 2018, 51(26): 265106.

[26] 张秋杰, 高占忠, 原玉, 等. 第一性原理研究荷电状态对 $\mathrm{Pd}_{13}$ 团 簇催化分解 NO 性能的影响. 原子与分子物理学报, 2016, 33(3): 438-442.

[27] JIA Q Q, JI H M, BAI X. Selective sensing property of triclinic $\mathrm{WO}_{3}$ nanosheets towards ultra-low concentration of acetone. Journal of Materials Science-Materials in Electronics, 2019, 30(8): 7824-7833.

[28] MA Y L, FENG B, LANG J Y, et al. Synthesis of semimetallic tungsten trioxide for infrared light photoelectrocatalytic water splitting. Journal of Physical Chemistry C, 2019, 123(42): 25833-25843.

[29] 秦京运, 舒群威, 袁艺, 等. $\mathrm{Ti}_{0.33} \mathrm{WO}_{3}$ 电子结构和太阳辐射屏蔽 性能第一性原理研究. 物理学报, 2020, 69(4): 047102.

[30] DIRAC P A M. The Principles of Quantum Mechanics. Oxford: Clarendon Press, 1958: 1-22.

[31] 曾瑾言. 量子力学. 北京, 科学出版社, 2000: 1-24.

[32] BORN M, HUANG K. Dynamical Theory of Crystal Lattices. Oxford: Oxford University Press, 1958: 104-113.

[33] SLATER J C. Magnetic effects and the Hartree-Fock equation. Physical Review, 1951, 82(4): 538-541.

[34] 张跃, 谷景华, 商家香, 等. 计算材料学基础. 北京: 北京航天 航空大学出版社, 2007: 83-135.

[35] 徐光宪, 黎乐民, 王德民. 量子化学: 基本原理和从头计算法. 北京: 科学出版社, 2007: 65-97.

[36] KOCH W, HOLTHAUSEN M C. A chemist's guide to density functional theory. Zeitschrift für Physik B Condensed Matter, 2001, 78(2): 317-323.

[37] KOHN W, SHAM L J. Self-consistent equations including exchange and correlation effects. Physical Review, 1965, 140: $1133-1138$.

[38] THOMAS H. The calculation of atomic fields. Proceedings of the Cambridge Philosophical Society, 1927, 23: 542-548.

[39] KOHN W. Nobel lecture: electronic structure of matter-wave functions and density functionals. Reviews of Modern Physics, 1999, 71(5): 1253-1266.

[40] FERMI E. Un metodo statistico per la determinazione di alcune Priorieta dell atome. Rendiconti Accademia Nazionale Dei Lincei, 1927, 6: 602

[41] DIRAC P A M. Note on exchange phenomena in the Thomas-Fermi atom. Proceedings of the Cambridge Philosophical Royal Society, 1930, 26: 376.

[42] SLATER J C. A simplification of the Hartree-Fock method. Self-Consistent Fields in Atoms, 1975, 81(3): 215-230.

[43] PERDEW J P, CHEVARY J A, VOSKO S H, et al. Atoms, molecules, solids, and surfaces-applications of the generalized gradient approximation for exchange and correlation. Physical Review B, 1992, 46(11): 6671-6687.

[44] 谢希德，陆栋. 固体能带理论. 上海: 复旦大学出版社, 1998: 66-69.

[45] VANDERBILT D. Soft self-consistent pseudopotentials in a generalized eigenvalue formalism. Physical Review B, 1990, 41(11): 7892-7895.

[46] BLÖCHL P E. Projector augmented-wave method. Physical Review B Condens Matter, 1994, 50(24): 17953-17959.

[47] HAMANN D R, SCHLÜTER M, CHIANG C. Norm-conserving pseudopotentials. Physical Review Letters, 1979, 43(20): 1494-1497.

[48] 北京创腾科技有限公司. BIOVIA materials studio. [EB/OL]. [2021-04-26] https://www.neotrident.com/index.php/product/proinfo/ 29.html.

[49] 北京宏剑公司. Vienna ab-initio simulation package. [EB/OL]. [2021-04-26] http://www.hongcam.com.cn/index.php/product/materials/ vasp.

[50] WEB V. Psi-k-Ab initio. [EB/OL]. [2021-04-26] http://psi-k.net/ software/.

[51] KAMINSKY W. WinXMorph. [EB/OL]. [2021-04-26] http://cad4.cpac. washington.edu/WinXMorphHome/WinXMorph.htm\#opennewwin dow.

[52] MOMMA K. Visualization for electronic and structural analysis. [EB/OL]. (2021-01-09) [2021-04-26] http://www.jp-minerals.org/ vesta/en/.

[53] MAHAJAN S, JAGTAP S. Metal-oxide semiconductors for carbon monoxide (CO) gas sensing: a review. Applied Materials Today, 2020, 18: 100483.

[54] ZEB S, PENG X J, YUAN G Z, et al. Controllable synthesis of ultrathin $\mathrm{WO}_{3}$ nanotubes and nanowires with excellent gas sensing performance. Sensors and Actuators B-Chemical, 2020, 305: 127435.

[55] LIU D, REN X W, LI Y S, et al. Nanowires-assembled $\mathrm{WO}_{3}$ nanomesh for fast detection of ppb-level $\mathrm{NO}_{2}$ at low temperature. Journal of Advanced Ceramics, 2020, 9(2): 17-26.

[56] OISON V, SAADI L, LAMBERT-MAURIAT C, et al. Mechanism of $\mathrm{CO}$ and $\mathrm{O}_{3}$ sensing on $\mathrm{WO}_{3}$ surfaces: first principle study. Sensors and Actuators B-Chemical, 2011, 160(1): 505-510.

[57] ZHAO L H, TIAN F H, WANG X B, et al. Mechanism of CO adsorption on hexagonal $\mathrm{WO}_{3}(001)$ surface for gas sensing: a DFT study. Computational Materials Science, 2013, 79: 691-697.

[58] JIN H, ZHOU H, ZHANG Y F. Insight into the mechanism of CO oxidation on $\mathrm{WO}_{3}(001)$ surfaces for gas sensing: a DFT study. Sensors, 2017, 17(8): 1898 .

[59] TANG B L, JIANG G H, CHEN W X, et al. First-principles study on hexagonal $\mathrm{WO}_{3}$ for $\mathrm{HCHO}$ gas sensing application. Acta Metallurgica Sinica-English Letters, 2015, 28(6): 772-780.

[60] HAN X, YIN X H. Density functional theory study of the $\mathrm{NO}_{2}$-sensing mechanism on a $\mathrm{WO}_{3}(001)$ surface: the role of surface oxygen vacancies in the formation of $\mathrm{NO}$ and $\mathrm{NO}_{3}$. 
Molecular Physics, 2016, 114(24): 3546-3555.

[61] QIN Y X, LIU M, HUA D Y. First-principles study of the electronic structure and $\mathrm{NO}_{2}$-sensing properties of Ti-doped $\mathrm{W}_{18} \mathrm{O}_{49}$ nanowire. Acta Physica Sinica, 2014, 63(20): 207101.

[62] QIN Y X, YE Z H. DFT study on interaction of $\mathrm{NO}_{2}$ with the vacancy-defected $\mathrm{WO}_{3}$ nanowires for gas-sensing. Sensors and Actuators B-Chemical, 2016, 222: 499-507.

[63] BAI S L, ZHANG K W, WANG L S, et al. Synthesis mechanism and gas-sensing application of nanosheet-assembled tungsten oxide microspheres. Journal of Materials Chemistry A, 2014, 2(21): 7927-7934.

[64] YAKOVKIN I N, GUTOWSKI M. Driving force for the $\mathrm{WO}_{3}(001)$ surface relaxation. Surface Science, 2007, 601(6): 1481-1488.

[65] 张克伟. 气敏和光催化导向的氧化铇纳米结构设计与改性. 北 京: 北京化工大学博士学位论文, 2014.

[66] YANG H H, SUN H G, LI Q T, et al. Structural, electronic, optical and lattice dynamic properties of the different $\mathrm{WO}_{3}$ phases: first-principle calculation. Vacuum, 2019, 164: 411-420.

[67] 杨欢欢. 第一性原理研究 $\mathrm{WO}_{3}$ 低指数表面上 $\mathrm{H}_{2} \mathrm{O}$ 分子吸附和分 解的微观机制. 济南: 山东大学硕士学位论文, 2019.

[68] ZHENG T T, SANG W, HE Z H, et al. Conductive tungsten oxide nanosheets for highly efficient hydrogen evolution. Nano Letters, 2017, 17(12): 7968-7973.

[69] 桑炜. 纳米晶可控合成以及催化性能的调控. 合肥: 中国科学 技术大学博士学位论文, 2018 .

[70] WANG F G, DI VALENTIN C, PACCHIONI G. Doping of $\mathrm{WO}_{3}$ for photocatalytic water splitting: hints from density functional theory. Journal of Physical Chemistry C, 2012, 116(16): 8901-8909.

[71] ZHANG T, ZHU Z L, CHEN H N, et al. Iron-doping-enhanced photoelectrochemical water splitting performance of nanostructured $\mathrm{WO}_{3}$ : a combined experimental and theoretical study. Nanoscale, 2015, 7(33): 2933-2940.

[72] HUANG W C, WANG J X, BIAN L, et al. Oxygen vacancy induces self-doping effect and metalloid LSPR in non-stoichiometric tungsten suboxide synergistically contributing to the enhanced photoelectrocatalytic performance of $\mathrm{WO}_{3-x} / \mathrm{TiO}_{2-x}$ heterojunction. Physical Chemistry Chemical Physics, 2018, 20(25): 17268-17278.

[73] ZHANG N, LI X Y, LIU Y F, et al. Defective tungsten oxide hydrate nanosheets for boosting aerobic coupling of amines: synergistic catalysis by oxygen vacancies and bronsted acid sites. Small, 2017, 13(31): 1701354.

[74] ZHANG N, JALIL A, WU D X, et al. Refining defect states in $\mathrm{W}_{18} \mathrm{O}_{49}$ by Mo doping: a strategy for tuning $\mathrm{N}_{2}$ activation towards solar-driven nitrogen fixation. Journal of the American Chemical Society, 2018, 140(30): 9434-9443.

[75] ZHANG N, LONG R, GAO C, et al. Recent progress on advanced design for photoelectrochemical reduction of $\mathrm{CO}_{2}$ to fuels. Science China-Materials, 2018, 61(6): 771-805.

[76] LI M Q, ZHANG N, LONG R, et al. PdPt alloy nanocatalysts supported on $\mathrm{TiO}_{2}$ : maneuvering metal-hydrogen interactions for light-driven and water-donating selective alkyne semihydrogenation. Small, 2017, 13(23): 1604173.

[77] WANG Z, WANG X Y, CONG S, et al. Fusing electrochromic technology with other advanced technologies: a new roadmap for future development. Materials Science \& Engineering R-Reports, 2020, 140: 100524.

[78] YAO Y J, ZHAO Q, WEI W, et al. $\mathrm{WO}_{3}$ quantum-dots electrochromism. Nano Energy, 2020, 68: 104350.

[79] LIN H, ZHOU F, LIU C P, et al. Non-grotthuss proton diffusion mechanism in tungsten oxide dihydrate from first-principles calculations. Journal of Materials Chemistry A, 2014, 2(31): 12280-12288.

[80] HJELM A, GRANQVIST C G, WILLS J M. Electronic structure and optical properties of $\mathrm{WO}_{3}, \mathrm{LiWO}_{3}, \mathrm{NaWO}_{3}$, and $\mathrm{HWO}_{3}$. Physical Review B, 1996, 54(4): 2436-2445.

[81] WISEMAN P J, DICKENS P G. Neutron-diffraction studies of cubic tungsten bronzes. Journal of Solid State Chemistry, 1976 , 17(1/2): 91-100.

[82] YANG S, CHA J, KIM J C, et al. Monolithic interface contact engineering to boost optoelectronic performances of $2 \mathrm{D}$ semiconductor photovoltaic heterojunctions. Nano Letters, 2020, 20(4): 2443-2451.

[83] 方城, 汪洪, 施思齐. 氧化铇电致变色性能的研究进展. 物理学 报, 2016, 65(16): 168201 .

[84] 高占忠. 锂离子电池负极材料 $\mathrm{WO}_{3}$ 的第一性原理研究. 成都: 电子科技大学硕士学位论文, 2017.

[85] KOCER C P, GRIFFITH K J, GREY C P, et al. Cation disorder and lithium insertion mechanism of Wadsley-Roth crystallographic shear phases from first principles. Journal of the American Chemical Society, 2019, 141(38): 15121-15134.

[86] KARIM N A, KAMARUDIN S K, SHYUAN L K, et al. Study on the electronic properties and molecule adsorption of $\mathrm{W}_{18} \mathrm{O}_{49}$ nanowires as a catalyst support in the cathodes of direct methanol fuel cells. Journal of Power Sources, 2015, 288: 461-472.

[87] ZHANG Z F, CHEN J L, LI H B, et al. Vapor-solid nanotube growth via sidewall epitaxy in an environmental transmission electron microscope. Crystal Growth \& Design, 2017, 17(1): 11-15.

[88] ZHANG Z F, WANG Y, LI H B, et al. Atomic-scale observation of vapor-solid nanowire growth via oscillatory mass transport. ACS Nano, 2016, 10(1): 763-769.

[89] ZHANG Z F, SHENG L P, CHEN L, et al. Atomic-scale observation of pressure-dependent reduction dynamics of $\mathrm{W}_{18} \mathrm{O}_{49}$ nanowires using environmental TEM. Physical Chemistry Chemical Physics, 2017, 19(25): 16307-16311.

[90] CHEN L, LAM S, ZENG Q H, et al. Effect of cation intercalation on the growth of hexagonal $\mathrm{WO}_{3}$ nanorods. Journal of Physical Chemistry C, 2012, 116(21): 11722-11727.

[91] JIANG S, CHEKINI M, QU Z B, et al. Chiral ceramic nanoparticles and peptide catalysis. Journal of the American Chemical Society, 2017, 139(39): 13701-13712.

[92] GU L J, MA C L, ZHANG X H, et al. Populating surface-trapped electrons towards SERS enhancement of $\mathrm{W}_{18} \mathrm{O}_{49}$ nanowires. Chemical Communications, 2018, 54(49): 6332-6335.

[93] MEHMOOD F, PACHTER R, MURPHY N R, et al. Effect of oxygen vacancies on the electronic and optical properties of tungsten oxide from first principles calculations. Journal of Applied Physics, 2016, 120(23): 233105.

[94] MIGAS D B, SHAPOSHNIKOV V L, RODIN V N, et al. Tungsten oxides. I. Effects of oxygen vacancies and doping on electronic and optical properties of different phases of $\mathrm{WO}_{3}$. Journal of Applied Physics, 2010, 108(9): 093713.

[95] SAI L W, TANG L L, HUANG X M, et al. Lowest-energy structures of $\left(\mathrm{WO}_{3}\right)_{n}(2 \leqslant n \leqslant 12)$ clusters from first-principles global search. Chemical Physics Letters, 2012, 544: 7-12.

[96] HUANG X, ZHAI H J, LI J, et al. On the structure and chemical bonding of tri-tungsten oxide clusters $\mathrm{W}_{3} \mathrm{O}_{n}{ }^{-}$and $\mathrm{W}_{3} \mathrm{O}_{n}(n=7-10)$ : $\mathrm{W}_{3} \mathrm{O}_{8}$ as a potential molecular model for O-deficient defect sites in tungsten oxides. Journal of Physical Chemistry A, 2006, 110(1): 85-92.

[97] 邱克强, 王爱民, 张海峰, 等. 铇丝增强 $\mathrm{ZrAlNiCuSi}$ 块体非晶 复合材料及其塑性行为. 金属学报, 2002(10): 1091-1096.

[98] JIANG P G, XIAO Y Y, LIU W J, et al. Hydrogen reduction characteristics of $\mathrm{WO}_{3}$ based on density functional theory. Results in Physics, 2019, 12: 896-902.

[99] LIU W J, JIANG P G, XIAO Y Y, et al. A study of the hydrogen adsorption mechanism of $\mathrm{W}_{18} \mathrm{O}_{49}$ using first-principles calculations. Computational Materials Science, 2018, 154: 53-59.

[100] 宋翰林, 姜平国, 刘文杰, 等. 氧化铇氢还原动力学的研究进展. 有色金属科学与工程, 2017, 8(5): 64-69.

[101] 姜平国, 汪正兵, 闵永播. 三氧化铇表面氢吸附机理的第一性 原理研究. 物理学报, 2017, 66(8): 080801 . 